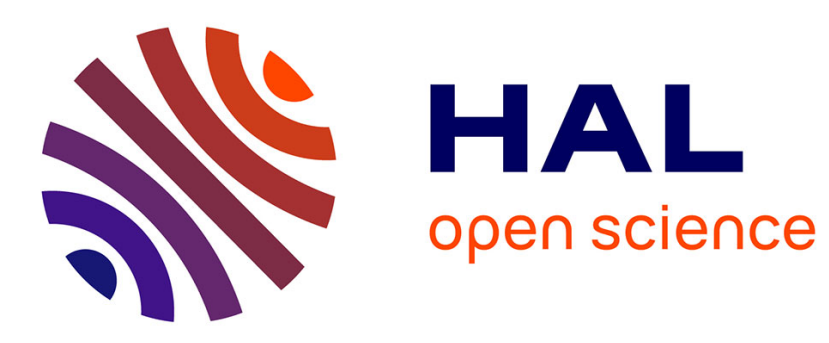

\title{
Efficient Enumeration of Non-Equivalent Squares in Partial Words with Few Holes
}

Panagiotis Charalampopoulos, Maxime Crochemore, Costas Iliopoulos, Tomasz Kociumaka, Solon P Pissis, Jakub Radoszewski, Wojciech Rytter, Tomasz Waleń

\section{To cite this version:}

Panagiotis Charalampopoulos, Maxime Crochemore, Costas Iliopoulos, Tomasz Kociumaka, Solon P Pissis, et al.. Efficient Enumeration of Non-Equivalent Squares in Partial Words with Few Holes. COCOON, Aug 2017, Hong Kong, China. hal-01787563

\section{HAL Id: hal-01787563 https://hal.science/hal-01787563}

Submitted on 7 May 2018

HAL is a multi-disciplinary open access archive for the deposit and dissemination of scientific research documents, whether they are published or not. The documents may come from teaching and research institutions in France or abroad, or from public or private research centers.
L'archive ouverte pluridisciplinaire HAL, est destinée au dépôt et à la diffusion de documents scientifiques de niveau recherche, publiés ou non, émanant des établissements d'enseignement et de recherche français ou étrangers, des laboratoires publics ou privés. 


\title{
Efficient Enumeration of Non-Equivalent Squares in Partial Words with Few Holes
}

\author{
Panagiotis Charalampopoulos ${ }^{1}$, Maxime Crochemore ${ }^{1,2}$, Costas S. Iliopoulos ${ }^{1}$, \\ Tomasz Kociumaka $^{3, \star}$, Solon P. Pissis ${ }^{1}$, Jakub Radoszewski ${ }^{1,3, \star \star}$, \\ Wojciech Rytter ${ }^{3, \star \star \star}$, and Tomasz Waleń ${ }^{3, \star \star \star}$ \\ 1 Department of Informatics, King's College London, London, UK \\ [panagiotis . charalampopoulos, maxime.crochemore, \\ costas.iliopoulos, solon.pissis] @kcl.ac.uk \\ 2 Université Paris-Est, France \\ 3 Faculty of Mathematics, Informatics and Mechanics, \\ University of Warsaw, Warsaw, Poland \\ [kociumaka, jrad, rytter, walen] @mimuw .edu.pl
}

\begin{abstract}
A partial word is a word with holes (also called don't cares: special symbols which match any symbol). A $p$-square is a partial word matching at least one standard square without holes (called a full square). Two p-squares are called equivalent if they match the same sets of full squares. Denote by psquares $(T)$ the number of non-equivalent p-squares which are subwords of a partial word $T$. Let $\operatorname{PSQUARES}_{k}(n)$ be the maximum value of psquares $(T)$ over all partial words of length $n$ with $k$ holes. We show asympthotically tight bounds:

$$
c_{1} \cdot \min \left(n k^{2}, n^{2}\right) \leq \operatorname{PSQUARES}_{k}(n) \leq c_{2} \cdot \min \left(n k^{2}, n^{2}\right)
$$

for some constants $c_{1}, c_{2}>0$. We also present an algorithm that computes psquares $(T)$ in $\mathcal{O}\left(n k^{3}\right)$ time for a partial word $T$ of length $n$ with $k$ holes. In particular, our algorithm runs in linear time for $k=\mathcal{O}(1)$ and its time complexity near-matches the maximum number of non-equivalent p-squares.
\end{abstract}

\section{Introduction}

A word is a sequence of letters from a given alphabet $\Sigma$. By $\Sigma^{*}$ we denote the set of all words over $\Sigma$. A word of the form $U U$, for some word $U$, is called a square. For a word $W$, a square factor is a factor of $W$ which is a square. Enumeration of square factors in words is a well-studied topic, both from a combinatorial and from an algorithmic perspective. Obviously, a word of length $n$ of may contain $\Theta\left(n^{2}\right)$ square factors (e.g. $\left.a^{n}\right)$, however, it is known that such a word contains

\footnotetext{
* Supported by Polish budget funds for science in 2013-2017 as a research project under the 'Diamond Grant' program.

$\star \star$ The author is a Newton International Fellow.

$\star \star \star$ Supported by the Polish National Science Center, grant no 2014/13/B/ST6/00770.
} 
only $\mathcal{O}(n)$ different square factors [16,20]; currently the best known upper bound is $\frac{11}{6} n$ [14]. Moreover, all different square factors of a word can be listed in $\mathcal{O}(n)$ time using two different approaches $[17,11]$.

A partial word is a sequence of letters from $\Sigma \cup\{\diamond\}$, where $\diamond$ denotes a hole, that is, a don't care symbol. Two symbols $a, b \in \Sigma \cup\{\diamond\}$ are said to match (denoted as $a \approx b$ ) if they are equal or one of them is a hole; note that this relation is not transitive. The relation of matching is extended in a natural way to partial words of the same length. A partial word $U V$ is called a $p$-square if $U \approx V$. We define the length of a p-square as $|U|$. Same as in the context of words, a $p$-square factor of a partial word $W$ is a factor being a p-square; see $[2,9]$. Alongside $[2,8,9]$, we define a full square as a square of a word and square subword of a partial word $W$ as a full square that matches some p-square factor of $W$.

We introduce the notion of equivalence of p-square factors in partial words. By $s q-v a l(U V)$ let us denote the set of different full squares that match the partial word $U V: \operatorname{sq}-\operatorname{val}(U V)=\left\{X X: X \in \Sigma^{*}, X X \approx U V\right\}$. Then p-squares $U V$ and $U^{\prime} V^{\prime}$ are called equivalent if $\operatorname{sq}$-val $(U V)=\operatorname{sq-val}\left(U^{\prime} V^{\prime}\right)$ (denoted as $\left.U V \equiv U^{\prime} V^{\prime}\right)$. E.g., the following two sets are equivalence classes of p-squares: $\mathcal{A}=\{a a \diamond \diamond, \diamond a a \diamond, \diamond \diamond a a, a \diamond \diamond a\}$ and $\mathcal{B}=\{a \diamond a \diamond \diamond \diamond, \diamond \diamond a a \diamond \diamond, \ldots\}$. Note that two psquare factors of a partial word $W$ are equivalent in this sense iff they correspond to exactly the same sets of square subwords. Our work is devoted to enumeration of non-equivalent p-square factors of a partial word with a given number $k$ of holes.

We say that $W W$ is a general form of a square $U V$ (see [8]), denoted as $\operatorname{repr}(U V)$, if $W W \approx U V$ and $\operatorname{sq-val}(W W)=\operatorname{sq}-\operatorname{val}(U V)$ (in other words, $W$ is the "most general" partial word that matches both $U$ and $V)$. Then $U V \equiv U^{\prime} V^{\prime}$ iff $\operatorname{repr}(U V)=\operatorname{repr}\left(U^{\prime} V^{\prime}\right)$. E.g., $\operatorname{repr}(A)=(a a)^{2}$ for all $A \in \mathcal{A}$ and $\operatorname{repr}(B)=$ $(a \diamond a)^{2}$ for all $B \in \mathcal{B}$.

Previous study of p-squares in partial words was mostly focused on their combinatorics. It started with the case of $k=1$ hole [8], in which case different square subwords correspond to non-equivalent p-square factors. It was shown that a partial word with one hole contains at most $\frac{7}{2} n$ different square subwords [6] (later this bound was improved to $3 n$ for binary partial words [19]). Also a generalization of the three squares lemma for words [12] was proposed for partial words [7].

As for a larger number of holes, the study was devoted mainly to counting the number of different square subwords of a partial word [8,2] or all occurrences of p-square factors [4,2]. Similarly, on the algorithmic side, [25] proved that the problem of counting different square subwords of a partial word is \#P-complete and $[15,24]$ and [9] showed quadratic- and nearly-quadratic-time algorithms for finding all occurrences of p-square factors and primitively-rooted p-square factors of a partial word, respectively. Other work includes avoidance of squares $[18,3]$ or abelian squares [5] in partial words. 


\subsection{Our Results}

We present the following combinatorial bounds and efficient algorithms related to enumeration of non-equivalent p-square factors of a partial word of length $n$ with $k$ of holes. A length $p$ is called ambiguous if there are two holes at a distance $p$. Otherwise it is called unambiguous.

Combinatorial results. We prove that a partial word of length $n$ with $k$ holes contains $\mathcal{O}\left(n k^{2}\right)$ non-equivalent p-squares. We also show an example of a partial word of length $n$ with $k$ holes that contains $\Omega\left(n k^{2}\right)$ non-equivalent psquares of ambiguous lengths and that contains $\Omega(n k)$ non-equivalent p-squares of unambiguous lengths. This work can be viewed as a generalization of the results on partial words with one hole $[8,6,19]$ to $k>1$ holes.

Algorithmic results. We present an algorithm that reports all non-equivalent p-squares in a partial word in $\mathcal{O}\left(n k^{3}\right)$ time. In particular, our algorithm runs in linear time for $k=\mathcal{O}(1)$ and its time complexity near-matches the maximum number of non-equivalent p-squares. Our algorithm generalizes the approach of [11] and proposes, as an important tool, a non-obvious extension of the notion of runs to partial words (another definition of runs in partial words appeared in $[9])$.

\section{Periodicity of Words and Partial Words}

A word $T$ is a sequence of letters over an alphabet $\Sigma$. By $|T|=n$ we denote the length of $T$, and by $T[i]$, for $i=1, \ldots, n$, the $i$ th letter of $T$. For $1 \leq i \leq j \leq n$, $T[i . . j]$ denotes the factor of $T$ equal to $T[i] \ldots T[j]$. A positive integer $q$ is called a period of $T$ if $T[i]=T[i+q]$ for all $i=1, \ldots, n-q$. In this case, $T[1 . . q]$ is called a string period of $T$. Two equal-length words $S$ and $T$ are called cyclic shifts if there exists an index $i$ such that $S[i . .|S|] S[1 . . i-1]=T$.

A run (also called a maximal repetition) in $T$ is a triple $(a, b, q)$ such that $T[a . . b]$ has the shortest period $q, 2 q \leq b-a+1$, and the interval cannot be extended to the left nor to the right without violating the above property, that is, $T[a-1] \neq T[a+q-1]$ and $T[b-q+1] \neq T[b+1]$, provided that the respective letters exist. The exponent of a run is defined as $\frac{b-a+1}{q}$. A word of length $n$ has at most $n$ runs and they can all be computed in $\mathcal{O}(n)$ time $[22,1]$.

From a run $(a, b, q)$ we can produce all triples $(a, b, k q)$ for integer $k$ such that $2 k q \leq b-a+1$; we call such triples generalized runs. In other words, the period specified in a generalized run need not be the shortest period of the fragment. The number of generalized runs is also $\mathcal{O}(n)$, as the sum of exponents of runs is $\mathcal{O}(n)[22,1]$.

A partial word is a sequence of symbols from $\Sigma^{\prime}=\Sigma \cup\{\diamond\}$. For a partial word $T$ we use the same notation as for words: $|T|$ for length, $T[i]$ for the $i$ th letter, $T[i . . j]$ for a factor. The relation of matching on $\Sigma^{\prime}$ is defined as $a \approx a$, $a \approx \diamond$ for all $a \in \Sigma^{\prime}$. We define an operation $\wedge$ such that $a \wedge a=a, a \wedge \diamond=a$ for 
all $a \in \Sigma^{\prime}$, and otherwise $a \wedge b$ is undefined. Two equal-length partial words $T$ and $S$ are said to match (denoted as $T \approx S$ ) if $T[i] \approx S[i]$ for all $i=1, \ldots,|T|$. In this case, by $S \wedge T$ we denote the partial word $S[1] \wedge T[1], \ldots, S[|S|] \wedge T[|S|]$. Note that if $U V$ is a p-square, then $\operatorname{repr}(U V)=(U \wedge V)^{2}$.

A quantum period of a partial word $T$ is a positive integer $q$ such that $T[i] \approx$ $T[i+q]$ for all $i=1, \ldots,|T|-q . T$ is called quantum periodic with a quantum period $q$ if $2 q \leq|T|$. Let $T$ be a partial word of length $n$. We say that a triple $(a, b, q)$ is a quantum generalized run (Q-run, for short) in $T$ if $T[a . . b]$ is quantum periodic with period $q$ and none of the partial words $T[a-1 . . b]$ and $T[a . . b+1]$ (if exists) has the quantum period $q$; see Example 1 and Fig. 2.

Example 1. The partial word $T=c a a \diamond \diamond \diamond \diamond \diamond b b d$ contains one Q-run with period 2: $(2,10,2)$ that corresponds to the factor $a a \diamond \diamond \diamond \diamond \diamond b b$.

Generalized runs in standard words are strongly related to squares: (1) every square belongs to a generalized run and, moreover, (2) all factors of length $2 q$ of a generalized run with period $q$ are squares being each other's cyclic shifts. Unfortunately, Q-runs in partial words have only property (1). However, we can introduce a type of run in partial words for which both properties (1) and (2) hold. A pseudorun (P-run, in short) is a triple $(a, b, q)$ such that:

(a) $T[a . . b]$ is quantum periodic with period $q$

(b) $T[i] \wedge T[i+q]=T[i+q] \wedge T[i+2 q]$ for all $i$ such that $i, i+2 q \in[a, b]$,

(c) none of the partial words $T[a-1 . . b]$ and $T[a . . b+1]$ (if exists) satisfies the conditions (a) and (b).

We say that a p-square $T[c . . d]$ is induced by the P-run if the length of the p-square is $q$ and $[c, d] \subseteq[a, b]$.

Observation 2. (1) Every p-square factor in $T$ is induced by a P-run. (1) All factors of length $2 q$ of a $P$-run with period $q$ are $p$-squares and their representatives are each other's cyclic shifts.

Proof. (1) Let $T[i . . j]$ be a p-square factor of length $q=(j-i+1) / 2$ in $T$. Initially we set $a=i, b=j$; then $(a, b, q)$ satisfies conditions (a) and (b) of a pseudorun (the latter one trivially). Now we extend $(a, b, q)$ until it becomes maximal under the two conditions, i.e., decrement $a$ while $T[a-1] \wedge T[a+q-1]=$ $T[a+q-1] \wedge T[a+2 q-1]$, and same for $b$.

(2) Every factor of length $2 q$ of a P-run is quantum periodic with period $q$, hence a p-square. Consider two such consecutive factors $X=T[i . . i+2 q-1]$ and $Y=T[i+1 . . i+2 q]$. Then $\operatorname{repr}(X)=T[i . . i+q-1] \wedge T[i+q . . i+2 q-1]$ and

$$
\begin{aligned}
\operatorname{repr}(Y) & =T[i+1 . . i+q] \wedge T[i+q+1 . . i+2 q] \\
& =T[i+1 . . i+q-1] \wedge T[i+q+1 . . i+2 q-1], T[i+q] \wedge T[i+2 q] \\
& =T[i+1 . . i+q-1] \wedge T[i+q+1 . . i+2 q-1], T[i] \wedge T[i+q]
\end{aligned}
$$

where the last equality is due to condition (b) of a P-run. Consequently, $\operatorname{repr}(X)$ and $\operatorname{repr}(Y)$ are cyclic shifts. 
Example 3. Let $T=c a a \diamond \diamond \diamond \diamond \diamond b b d$. Then $T$ contains five P-runs with period 2: $(2,5,2),(3,6,2),(4,8,2),(6,9,2)$ and $(7,10,2)$, that correspond to factors: $a a \diamond \diamond$, $a \diamond \diamond \diamond, \diamond \diamond \diamond \diamond \diamond, \diamond \diamond \diamond b$, and $\diamond \diamond b b$, respectively. The squares induced by the respective P-runs are as follows: $a a \diamond \diamond ; a \diamond \diamond \diamond ; \diamond \diamond \diamond \diamond$ (two times); $\diamond \diamond \diamond b$; and $\diamond \diamond b b$.

\section{Combinatorial Bounds}

Theorem 4. There exists a partial word of length $n$ with $k$ holes that contains $\Omega\left(n k^{2}\right)$ non-equivalent $p$-squares, for $k=\mathcal{O}(\sqrt{n})$.

Proof. Assume that $2 \mid k$. Consider the following partial word:

$$
T_{m, k}=a^{m} \diamond^{k / 2} a^{m}\left(a^{k / 2-1} \diamond\right)^{k / 2} a^{3 m}
$$

of length $n=4 m+\left(\frac{k}{2}\right)^{2}+\frac{k}{2}$ over $\Sigma=\{a, b\}$; here $m=\Theta(n)$. Point (a) follows from the next claim.

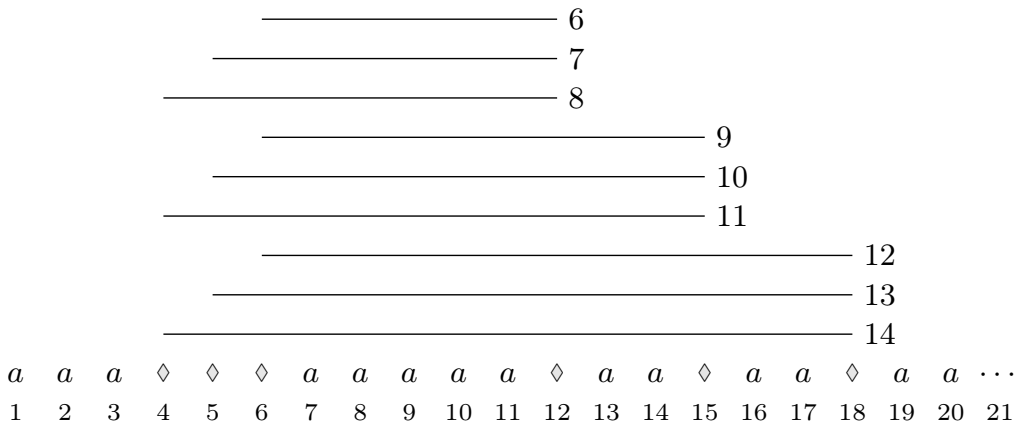

Fig. 1. Ambiguous lengths in $T_{3,6}$.

Claim. For some $k=\mathcal{O}(\sqrt{n}), T_{m, k}$ contains $\Theta\left(m k^{2}\right)$ non-equivalent p-squares of ambiguous lengths.

Proof. First, note that that there are $\left(\frac{k}{2}\right)^{2}$ different ambiguous lengths and that for each such length $\ell$, there is exactly one pair of holes at this distance in $T_{m, k}$. Indeed, consider the $i$-th hole in the $\diamond^{k / 2}$ part and the $j$-th hole in the $\left(a^{k / 2-1} \diamond\right)^{k / 2}$ part $\left(1 \leq i, j \leq \frac{k}{2}\right)$. Then the distance between them is $\frac{k}{2}-i+$ $m+\frac{k}{2} \cdot j=\frac{k}{2} \cdot j-i+\left(\frac{k}{2}+m\right)$. Finally, note that for $i$ and $j$ in the considered range, values of the form $\frac{k}{2} \cdot j-i$ are all distinct (this is a known example of a differenece cover); see Fig. 1.

Let us choose $k$ so that $2\left(m+\left(\frac{k}{2}\right)^{2}+\frac{k}{2}\right) \leq \frac{n}{2}$. Then for each ambiguous length $\ell$, we have exactly $\ell$ p-square factors in $T_{m, k}$ of the general form $\left(a^{i} \diamond a^{\ell-i-1}\right)$. As $\ell \geq m=\Theta(n)$, this concludes the proof. 
(b) $\ldots$

Now we proceed to the upper bound proof. Let us fix a partial word $T$ of length $n$ with $k$ holes. Let holes $(a, b)$ denote the number of holes in $T[a . . b]$.

We say that a square subword has a solid occurrence if... By the following fact, there are $\mathcal{O}(n)$ square subwords of $T$ with solid occurrences.

Fact $5([\mathbf{1 6}, \mathbf{2 0}, \mathbf{1 4}]) \cdot \leq 2$ rightmost occurrences.

Our upper bound for partial words is based on the following key lemma that generalizes Fact 5. We call an interval $[\ell, r) \subseteq[1, n]$ is short if $r \leq \frac{3 k+3}{3 k+2} \ell$.

Lemma 6. For a short interval $[\ell, r)$, there are $\mathcal{O}\left(k^{\prime}\right)$, where $k^{\prime}=$ holes $(i, i+$ $2 r-1), p$-squares of unambiguous lengths without solid occurrences in $[\ell, r]$ that have their rightmost occurrence in $T$ at position $i$.

Proof. Let us start with the following claim.

Claim 7. There exists an interval $I=[a, b] \subseteq[i, i+\ell-1]$ such that holes $(I)=0$, holes $(J)=0$ where $J=[a+\ell, b+r)$, and $I \geq 2(r-\ell)$.

Proof. A position $i$ cannot belong to $I$ if $T[i]$ is a hole or $T[i+\ell . . i+r]$ contains a hole. In total, a hole in $T[i . . i+\ell-1]$ excludes one positions in $[i, i+\ell-1]$ and a hole in $T[i+\ell . . i+2 r-1]$ excludes at most $r-\ell+1$ consecutive positions in $[i, i+\ell-1]$. By the pigeonhole principle, there exists a non-excluded fragment in $T[i . . i+\ell-1]$ of consecutive positions of length at least

$$
\frac{\ell-k(r-l)}{k+1} \geq \frac{(3 k+2)(r-\ell)-k(r-l)}{k+1}=2(r-\ell) .
$$

Let $X$ be the subword of $T$ at positions in $I$. If there are at least two p-square factors of the considered type starting at position $i$, then $X$ is periodic (Galil...). Let $p$ be its shortest period.

Let $U V$ be a p-square factor of unambiguous length of the considered type and let $W W=\operatorname{repr}(U V)$ There are three cases:

(a) $W W$ has period $p$

(b) $W$ has period $p$

(c) $W$ does not have period $p$.

There is at most one p-square factor $U V$ corresponding to case (a). Assume to the contrary that there are two such p-squares, $U V$ and $U^{\prime} V^{\prime}$, of lengths $d<d^{\prime}$. We have that $p \mid d, d^{\prime}$ and the generalized forms of the two p-squares have the same string period (as they share a subword $U$ ). Hence, $\operatorname{repr}(U V)$ is a border of $\operatorname{repr}\left(U^{\prime} V^{\prime}\right)$, so $U V$ occurs in $T$ at position $i+d^{\prime}-d$.

We will show that there are at most $k^{\prime}+1$ p-square subwords of type (b). Assume to the contrary that at least $k^{\prime}+2$ of them, of lengths $d_{1}<\ldots<d_{k^{\prime}+2}$. We see that $d_{j} \bmod k$ is the same, as the occurrences of $X$ in the right half of the 
p-square in $T$ differ by multiples of $p$. Consider the shortest $U V$ and the longest $U^{\prime} V^{\prime}$ these p-squares, with generalized forms $W W$ and $W^{\prime} W^{\prime}$, and the subword $T\left[i+d_{1} . . i+d_{k^{\prime}+2}-1\right]$. It matches a prefix $P$ of length $d_{k^{\prime}+2}-d_{1}$ of $W$ and a suffix $S$ of the same length of $W^{\prime}$. Both $P$ and $S$ have period $P$; however, their string periods are not equal. Consequently, in every occurrence of the period in $T\left[i+d_{1} . . i+d_{k^{\prime}+2}-1\right]$ there mus be a hole; this makes $\left(d_{k^{\prime}+2}-d_{1}\right) / p \geq k^{\prime}+1$ holes in total, a contradiction.

(c) Consider the occurrence of $X$ in $W$. The periodicity of $X$ does not cover the whole $W$, so there is a position $j$ in $W$ where the periodicity breaks. Assume w.l.o.g. that $j$ is to the right of $X$. Consider the positions $j_{1}$ and $j_{2}$ that correspond to $j$ in the subwords $U, V$ of $T$. If any of $T\left[j_{1}\right]$ and $T\left[j_{2}\right]$ is not a hole, then it is determined uniquely as the first position where the deterministic period $p$ breaks, starting from the corresponding occurrence of $X$. Hence, if both of them are not holes, then $|W|=j_{2}-j_{1}$ is uniquely determined. Otherwise, if $T\left[j_{1}\right]$ or $T\left[j_{2}\right]$ is a hole (they cannot be both holes, as the length is unambiguous), then one of $j_{1}, j_{2}$ is uniquely determined and there are at most $k^{\prime}$ choices for the other. Consequently, there are at most $4 k^{\prime}+2$ such p-squares.

Theorem 8. A partial word $T$ of length $n$ with $k$ holes contains $\mathcal{O}\left(n k^{2}\right)$ nonequivalent p-squares.

Proof. Obviously, in $T$ there are at most $k^{2}$ ambiguous lengths. Consequently, there are $\mathcal{O}\left(n k^{2}\right)$ non-equivalent p-squares of such lengths. Let us consider psquares of unambiguous lengths. By Fact 5 , among them there are $\mathcal{O}(n)$ different p-squares with a solid occurrence. From now on we consider only p-squares without a solid occurrence.

Let $[\ell, r]$ be a short interval. By Lemma 6 , the total number of different p-squares of unambiguous lengths in $[\ell, r]$ in $T$ is:

$$
\mathcal{O}\left(\sum_{i=1}^{n} k_{i, i+2 r-1}\right)=\mathcal{O}(r k) \text {. }
$$

The equality is based on the fact that each of the $k$ holes in $T$ is counted in at most $2 r$ terms $k_{i, i+2 r-1}$.

Let us consider a family of endpoints $r_{0}, r_{1}, \ldots$ :

$$
r_{j}=\left\lfloor\frac{n}{\left(1+\frac{1}{3 k+2}\right)^{j}}\right\rfloor=\left\lfloor n\left(\frac{3 k+2}{3 k+3}\right)^{j}\right\rfloor .
$$

We divide the p-square lengths into short intervals $\left[r_{j+1}+1, r_{j}\right]$. By (1), the total number of p-squares in $T$ is:

$$
\mathcal{O}\left(\sum_{j=0}^{\infty} r_{j} k\right)=\mathcal{O}\left(n k \sum_{j=0}^{\infty}\left(\frac{3 k+2}{3 k+3}\right)^{j}\right)=\mathcal{O}\left(\frac{n k}{1-\frac{3 k+2}{3 k+3}}\right)=\mathcal{O}\left(n k^{2}\right) .
$$




\section{Main Algorithm}

Let $T$ be a partial word of length $n$ with $k$ holes.

\subsection{Computing Q-runs}

We divide Q-runs into solid Q-runs that do not contain a hole and the remaining non-solid Q-runs. A solid Q-run is a generalized run in a maximal solid factor of $T$. Thus all solid Q-runs can be computed in $\mathcal{O}(n)$ time via a linear-time algorithm for computing runs in words $[22,1]$.

Non-solid Q-runs are computed with a modification of Main-Lorentz algorithm [23]. Let us first reformulate the algorithm in the language of computing generalized runs in words. For a word $S$ of length $n$, it finds a representation of all p-squares that contain the position $i=\lfloor n / 2\rfloor$ and then makes recursive computations in $S[1 . . i]$ and $S[i . . n]$. It first computes all the p-squares with first half containing the position $i$ and then computes the remaining p-squares containing the position $i$ symmetrically. For a pair of positions $i, j$, we define $l c p(i, j)$ as the length of the longest common prefix of $S[i . . n]$ and $S[j . . n]$ and $l c s(i, j)$ as the length of the longest common suffix of $S[1 . . i]$ and $S[1 . . j]$. The algorithm for each position $j>i$ computes $l c p(i, j)+l c s(i, j)$ and, if this value is at least $j-i$, reports a generalized run $(i-l c s(i, j) . . j+l c p(i, j), j-i)$. To avoid duplicates, the generalized run can be omitted if it reaches the end of the word in the recursive call which does not coincide with the position 1 or $n$ in the original word. The algorithm's running time is $\mathcal{O}(n \log n)$ as $l c p$-queries and $l c s$-queries can be answered in $\mathcal{O}(1)$-time after preprocessing.

For a partial word $T$, the longest common compatible prefix of two positions $i, j$, denoted $l c c p(i, j)$, is defined as the largest $\ell$ such that $T[i . . i+\ell-1] \approx T[j . . j+$ $\ell-1]$. Symmetrically, we can define lccs as the length of the longest common compatible suffix. In [10] it was shown that after $\mathcal{O}(n k)$-time preprocessing, lccp-queries (hence, lccs-queries) can be answered in $\mathcal{O}(1)$ time.

Thus we could directly apply the Main-Lorentz scheme for partial words; the result would be exactly the set of Q-runs in $T$. However, this would yield $\mathcal{O}(n \log n)$-time computation. We aim only at computing non-solid Q-runs, which lets us easily reduce the complexity to $\mathcal{O}(n \log k)$. To this end, we only make recursive calls in positions that contain holes, with the recursive call taking

place at the position of the $\left\lceil\frac{k}{2}\right\rceil$-th hole in $T$. Together with $\mathcal{O}(n)$-time solid Q-runs computation we arrive at the following.

Lemma 9. A partial word of length $n$ with $k$ holes contains $\mathcal{O}(n \log k)$ Q-runs and they can all be computed in $\mathcal{O}(n \log k)$ time.

\subsection{Computing Pseudoruns}

Observation 10. If $(a, b, p)$ is a P-run, then there exists a $Q$-run $\left(a^{\prime}, b^{\prime}, p\right)$ such that $a^{\prime} \leq a \leq b \leq b^{\prime}$. 
From the set of $m$ Q-runs of period $p$ we can produce the set of all P-runs with period $p$. The computation time is $\mathcal{O}(m+k)$.

Lemma 11. A partial word of length $n$ with $k$ holes contains $\mathcal{O}(n k)$ P-runs and they can all be computed in $\mathcal{O}(n k)$ time.

\subsection{Reporting Squares}

Assume that the alphabet $\Sigma$ is ordered and that $\diamond$ is smaller than all the letters from $\Sigma$. We use Observation 2. If we group the P-runs by the minimal cyclic shift of the induced p-squares, then we can apply the approach of [11] to count the p-squares in time linear in the number of P-runs and $n$.

First, note that the minimal cyclic shift value for a P-run $(a, b, p)$ can be computed in $\mathcal{O}\left(k^{2}\right)$ time using Theorem 23 from [21]. Indeed, we can represent the representative of the p-square $T[a . . a+2 p-1]$ as a concatenation of at most $k$ factors of $T$ and at most $k$ single letters that appear elsewhere in $T$.

$\ldots$

Theorem 12. All non-equivalent $p$-squares in a partial word of length $n$ with $k$ holes can be reported (as factors of the partial word) in $\mathcal{O}\left(n k^{3}\right)$ time.

\section{References}

1. Bannai, H., I, T., Inenaga, S., Nakashima, Y., Takeda, M., Tsuruta, K.: A new characterization of maximal repetitions by Lyndon trees. CoRR abs/1406.0263 (2014), http://arxiv.org/abs/1406.0263

2. Blanchet-Sadri, F., Bodnar, M., Nikkel, J., Quigley, J.D., Zhang, X.: Squares and primitivity in partial words. Discrete Applied Mathematics 185, 26-37 (2015), http://dx.doi.org/10.1016/j.dam.2014.12.003

3. Blanchet-Sadri, F., Choi, I., Mercas, R.: Avoiding large squares in partial words. Theor. Comput. Sci. 412(29), 3752-3758 (2011), http://dx.doi.org/10.1016/j. tcs.2011.04.009

4. Blanchet-Sadri, F., Jiao, Y., Machacek, J.M., Quigley, J.D., Zhang, X.: Squares in partial words. Theor. Comput. Sci. 530, 42-57 (2014), http://dx.doi.org/10. 1016/j.tcs. 2014.02.023

5. Blanchet-Sadri, F., Kim, J.I., Mercas, R., Severa, W., Simmons, S.: Abelian squarefree partial words. In: Dediu et al. [13], pp. 94-105, http://dx.doi.org/10.1007/ 978-3-642-13089-2_8

6. Blanchet-Sadri, F., Mercas, R.: A note on the number of squares in a partial word with one hole. ITA 43(4), 767-774 (2009), http://dx.doi.org/10.1051/ ita/2009019

7. Blanchet-Sadri, F., Mercas, R.: The three-squares lemma for partial words with one hole. Theor. Comput. Sci. 428, 1-9 (2012), http://dx.doi.org/10.1016/j . tcs.2012.01.012

8. Blanchet-Sadri, F., Mercas, R., Scott, G.: Counting distinct squares in partial words. Acta Cybern. 19(2), 465-477 (2009), http://www.inf.u-szeged.hu/ actacybernetica/edb/vol19n2/BlanchetSadri_2009_ActaCybernetica.xml 
9. Blanchet-Sadri, F., Nikkel, J., Quigley, J.D., Zhang, X.: Computing primitivelyrooted squares and runs in partial words. In: Kratochvíl, J., Miller, M., Froncek, D. (eds.) Combinatorial Algorithms - 25th International Workshop, IWOCA 2014, Duluth, MN, USA, October 15-17, 2014, Revised Selected Papers. Lecture Notes in Computer Science, vol. 8986, pp. 86-97. Springer (2014), http://dx.doi.org/ 10.1007/978-3-319-19315-1_8

10. Crochemore, M., Iliopoulos, C.S., Kociumaka, T., Kubica, M., Langiu, A., Radoszewski, J., Rytter, W., Szreder, B., Waleń, T.: A note on the longest common compatible prefix problem for partial words. J. Discrete Algorithms 34, 49-53 (2015), http://dx.doi.org/10.1016/j.jda.2015.05.003

11. Crochemore, M., Iliopoulos, C.S., Kubica, M., Radoszewski, J., Rytter, W., Waleń, T.: Extracting powers and periods in a word from its runs structure. Theor. Comput. Sci. 521, 29-41 (2014), http://dx.doi.org/10.1016/j.tcs.2013.11.018

12. Crochemore, M., Rytter, W.: Sqares, cubes, and time-space efficient string searching. Algorithmica 13(5), 405-425 (1995), http://dx.doi.org/10.1007/ BF01190846

13. Dediu, A., Fernau, H., Martín-Vide, C. (eds.): Language and Automata Theory and Applications, 4th International Conference, LATA 2010, Trier, Germany, May 24-28, 2010. Proceedings, Lecture Notes in Computer Science, vol. 6031. Springer (2010), http://dx.doi.org/10.1007/978-3-642-13089-2

14. Deza, A., Franek, F., Thierry, A.: How many double squares can a string contain? Discrete Applied Mathematics 180, 52-69 (2015), http://dx.doi.org/10.1016/ j.dam.2014.08.016

15. Diaconu, A., Manea, F., Tiseanu, C.: Combinatorial queries and updates on partial words. In: Kutylowski, M., Charatonik, W., Gebala, M. (eds.) Fundamentals of Computation Theory, 17th International Symposium, FCT 2009, Wroclaw, Poland, September 2-4, 2009. Proceedings. Lecture Notes in Computer Science, vol. 5699, pp. 96-108. Springer (2009), http://dx.doi.org/10.1007/978-3-642-03409-1_ 10

16. Fraenkel, A.S., Simpson, J.: How many squares can a string contain? J. Comb. Theory, Ser. A 82(1), 112-120 (1998), http://dx.doi.org/10.1006/jcta.1997. 2843

17. Gusfield, D., Stoye, J.: Linear time algorithms for finding and representing all the tandem repeats in a string. J. Comput. Syst. Sci. 69(4), 525-546 (2004)

18. Halava, V., Harju, T., Kärki, T.: Square-free partial words. Inf. Process. Lett. 108(5), 290-292 (2008), http://dx.doi.org/10.1016/j.ipl.2008.06.001

19. Halava, V., Harju, T., Kärki, T.: On the number of squares in partial words. RAIRO - Theor. Inf. and Applic. 44(1), 125-138 (2010), http://dx.doi.org/10. $1051 /$ ita/2010008

20. Ilie, L.: A simple proof that a word of length $n$ has at most $2 n$ distinct squares. J. Comb. Theory, Ser. A 112(1), 163-164 (2005), http://dx.doi.org/10.1016/j. jcta.2005.01.006

21. Kociumaka, T.: Minimal suffix and rotation of a substring in optimal time. In: Grossi, R., Lewenstein, M. (eds.) 27th Annual Symposium on Combinatorial Pattern Matching, CPM 2016, June 27-29, 2016, Tel Aviv, Israel. LIPIcs, vol. 54, pp. 28:1-28:12. Schloss Dagstuhl - Leibniz-Zentrum fuer Informatik (2016), http://dx.doi.org/10.4230/LIPIcs.CPM.2016.28

22. Kolpakov, R.M., Kucherov, G.: Finding maximal repetitions in a word in linear time. In: 40th Annual Symposium on Foundations of Computer Science. pp. 596604. FOCS'99, IEEE Computer Society (1999) 
23. Main, M.G., Lorentz, R.J.: An $\mathrm{O}(\mathrm{n} \log \mathrm{n})$ algorithm for finding all repetitions in a string. J. Algorithms 5(3), 422-432 (1984), http://dx.doi.org/10.1016/ 0196-6774 (84) 90021-X

24. Manea, F., Mercas, R., Tiseanu, C.: An algorithmic toolbox for periodic partial words. Discrete Applied Mathematics 179, 174-192 (2014), http://dx.doi.org/ $10.1016 / \mathrm{j}$. dam.2014.07.017

25. Manea, F., Tiseanu, C.: Hard counting problems for partial words. In: Dediu et al. [13], pp. 426-438, http://dx.doi.org/10.1007/978-3-642-13089-2_36 


\section{A Auxiliary Figures}

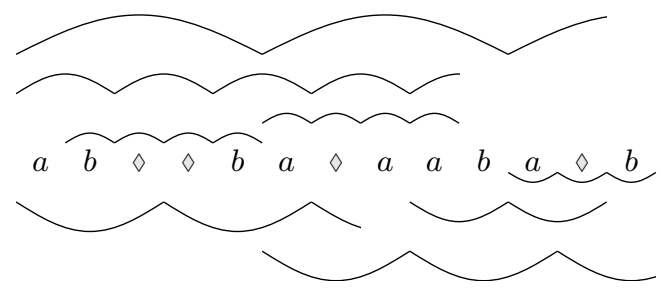

Fig. 2. A partial word together with all its Q-runs. This partial word contains 5 p-squares of length 3: $a a b a \diamond b, \diamond a a b a \diamond, a b \diamond \diamond b a, a \diamond a a b a, b \diamond \diamond b a \diamond$. The following pair is equivalent: $a b \diamond \diamond b a \equiv a \diamond a a b a$. 